\title{
Bewegungsapparat-Kurse und Lehrbücher auf irheuma.com publiziert
}

Sie finden unter http://www.irheuma.com/ \#books eine aktualisierte Liste von Lehrbüchern und von angebotenen Ultraschallkursen in der Schweiz und im Ausland.

Übersicht über die von QIR (www.qir.ch) anerkannten Fortbildungskurse (Stand 28.6.2020). Die Kurse sind SGUM-anerkannt!

- XIV and XV Interventional Musculoskeletal Ultrasound Cadaver Course,

Location: University of Basel, Anatomy Institute, Date: 13.8.2020
- „Sono-Sunday“ - Baseler Supervisionskurs, Location: Universitätsspital Basel, Date: 23.8.2020-6.9.2020

- EFSUMB endorsed VIII th Ultrasound Guided Interventional Cadaver Course, Location: University of Basel, Anatomy Institute, Date: 22.10.2020

- $2^{\text {th }}$ Musculoskeletal Ultrasound Refresher Course, Location: Bozen, Date: 16.-18.11.2020

- Ultraschall-Tage Appenzell, Location: Weissbad, Date: 6.-8.5.2021
- IX. Interventional cadaver course fluoroscopy and ultrasound guided joint and spine interventions, Location: University of Basel, Anatomical Institute, Date: 25.3.2021

- EFSUMB-2-CREDITS: 1. CME UZR Online-Course, Start: 1.7.2020: https://vimeo.com/uzr 